\title{
Three-dimensional Fourier domain optical coherence tomography in vivo imaging of alveolar tissue in the intact thorax using the parietal pleura as a window
}

\author{
Sven Meissner \\ University of Technology Dresden \\ Medical Faculty \\ Clinical Sensoring and Monitoring \\ Fetscherstrasse 74 \\ Dresden, 01307 Germany
}

Lilla Knels

University of Technology Dresden Medical Faculty

Clinic of Anesthesiology and Intensive Care Fetscherstrasse 74

Dresden, 01307 Germany

\section{Christian Schnabel}

University of Technology Dresden Medical Faculty

Clinical Sensoring and Monitoring

Fetscherstrasse 74

Dresden, 01307 Germany

\section{Thea Koch}

University of Technology Dresden

Medical Faculty

Clinic of Anesthesiology and Intensive Care

Fetscherstrasse 74

Dresden, 01307 Germany

\section{Edmund Koch}

University of Technology Dresden

Medical Faculty

Clinical Sensoring and Monitoring

Fetscherstrasse 74

Dresden, 01307 Germany

\begin{abstract}
In vivo determination of 3-D and dynamic geometries of alveolar structures with adequate resolution is essential for developing numerical models of the lung. A thorax window is prepared in anesthetized rabbits by removal of muscle tissue between the third and fourth rib without harming the parietal pleura. The transparent parietal pleura allows contact-free imaging by intravital microscopy (IVM) and 3-D optical coherence tomography (3-D OCT). We demonstrate that dislocation of the lung surface is small enough to observe identical regions in the expiratory and inspiratory plateau phase, and that OCT in this animal model is suitable for generating 3-D geometry of in vivo lung parenchyma. To our knowledge, we present a novel thorax window preparation technique for 3-D imaging of alveolar dynamics for the first time. The 3-D datasets of the fine structure of the lung beneath the pleura could provide a basis for the development of 3-D numerical models of the lung. () 2010 Society of Photo-Optical Instrumentation Engineers. [DOI: 10.1117/1.3302809]
\end{abstract}

Keywords: thorax window; alveolar dynamics; optical coherence tomography; alveolar geometry.

Paper 09417LRR received Sep. 16, 2009; revised manuscript received Jan. 6, 2010; accepted for publication Jan. 6, 2010; published online Feb. 9, 2010.

\section{Introduction}

Artificial ventilation is a life-saving therapy in respiratory failure, which at the same time can induce lung injury or impair lung function. ${ }^{1}$ The development of protective ventilatory strategies aims to decrease the incidence of ventilatorinduced lung injury. ${ }^{2}$ One aid in the development of new protective ventilation strategies is the generation of numerical models of the lung, requiring detailed information on alveolar dynamics during ventilation. However, in vivo dynamic 3-D geometries with adequate resolution have not yet been described in the literature. Hence, most scientists dealing with lung modeling use 2-D intravital microscopy (IVM) images or the results of histological examinations of fixed lung tissue as

Address all correspondence to: Sven Meissner, University of Technology Dresden, Medical Faculty, Clinical Sensoring and Monitoring, Fetscherstrasse 74 Dresden, 01307 Germany. Tel: 49-351-458-6133; Fax: 49-351-458-6325; E-mail: sven.meissner@tu-dresden.de. a basis for their numerical models, even though these do not describe a 3-D in vivo geometry. ${ }^{3}$ One imaging method for closing the lack of information could be Fourier domain optical coherence tomography (FD-OCT), which is a contactfree and high-speed imaging modality. ${ }^{4-6}$ FD-OCT allows 3-D imaging even in strong scattering samples such as biological tissue, with a resolution of less than $10 \mu \mathrm{m}$. FD-OCT is particularly suitable for performing investigations of lung tissue of isolated lungs through a thorax window in an in vivo mouse model, as described by our group in previous studies. ${ }^{7-9}$ To avoid pneumothorax and lung injury during preparation, we carried out a thorax window preparation without harming the parietal pleura. A similar preparation was already described by Wearn et al. ${ }^{10}$ for the visualization of pulmonary blood flow. OCT imaging was performed through the thorax window to provide 3-D geometry of subpleural alveolar structures

$1083-3668 / 2010 / 15(1) / 016030 / 4 / \$ 25.00$ @ $2010 \mathrm{SPIE}$ 
Meissner et al.: Three-dimensional Fourier domain optical coherence tomography in vivo imaging...

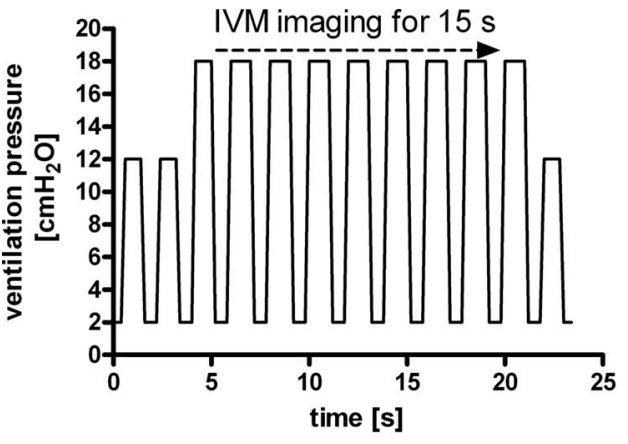

(a)

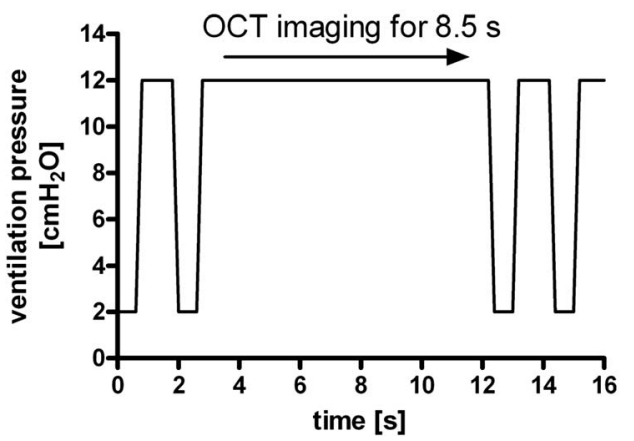

(b)

Fig. 1 Ventilation pressure during image acquisition by IVM (a) and OCT (b). The IVM was documented by a movie of $15 \mathrm{~s}$ focused either to the inspiratory $\left(18 \mathrm{cmH}_{2} \mathrm{O}\right)$ or the expiratory $\left(2 \mathrm{cmH}_{2} \mathrm{O}\right)$ plateau. I (b), the ventilation chart for OCT image acquisition for an applied CPAP of $12 \mathrm{cmH}_{2} \mathrm{O}$ is displayed. The inspiratory plateau is increased up to $10 \mathrm{~s}$ to provide a stable situation for 3-D OCT imaging for the required 8.5-s acquisition time. Before and after OCT imaging, the rabbit is regularly ventilated for at least $5 \mathrm{~min}$ to provide sufficient ventilation.

needed for developing numerical models of the lung.

\section{Methods and Materials}

\subsection{Window Preparation}

All experiments were performed in accordance with the Guide for the Care and Use of Laboratory Animals (7th edition, Institute of Laboratory Animal Resources, Washington, D.C., 1996). The study was approved by the animal care and use committee of the local government authorities (AZ: 24D9168.11-1/2008-29). Briefly, male rabbits weighing 2.5 to $3.0 \mathrm{~kg}$ were used in this feasibility study. After cannulation of the marginal ear vein, initial anesthesia was performed with ketamine $5-\mathrm{mg} / \mathrm{kg}$ bodyweight (bw) and xylazine $0.8 \mathrm{mg} / \mathrm{kg}$. The rabbits were tracheotomized and connected to a small animal respirator to provide pressurecontrolled ventilation with room air. Anesthetic depth was maintained by continuous injection of $10-\mathrm{mg} / \mathrm{h}$ ketamine and 3-mg/h xylazine. During preparation, the following ventilator settings were applied: 30 breaths per minute (bpm), inspiratory plateau pressure (IPP) of $10 \mathrm{cmH}_{2} \mathrm{O}$, and a positive endexpiratory pressure (PEEP) of $2 \mathrm{cmH}_{2} \mathrm{O}$ resulting in a tidal volume of approximately $8-\mathrm{ml} / \mathrm{kg}$ bw. Blood pressure was monitored by intra-arterial catheter in the ear artery. The rabbit was fixed on the operation table in a lateral position for preparation and image acquisition. The operating field was clipped and shaved. The skin was surgically opened with a 5-cm-long transcison and the upper intercostal muscle layers were resected between the third and fourth rib. The lower layers of muscle tissue were abraded using small forceps, while all muscle fibers overlying the parietal pleura were abscised in a region of approximately $10 \times 5 \mathrm{~mm}^{2}$. The resection of the lower muscle fibers has to be carried out very carefully to avoid injury of the pleura and lung tissue, pneumothorax, or mechanical stress during preparation. Blood for blood gas analysis was taken from the ear artery after preparation and at the end of the investigation.

\subsection{Image Acquisition}

Imaging through the prepared thorax window was achieved using 2-D intravital microscopy and 3-D FD-OCT. A newly developed scanner head combining both modalities was used. ${ }^{6,8}$ The subpleural alveolar structures were imaged using IVM, applying an IPP of $18 \mathrm{cmH}_{2} \mathrm{O}$ corresponding to a tidal volume of approximately $14-\mathrm{ml} / \mathrm{kg}$ bw. A first image series of $15 \mathrm{~s}$ was taken focused on the inspiratory plateau (see Fig. 1). After $5 \mathrm{~min}$ of regular ventilation (IPP $12 \mathrm{cmH}_{2} \mathrm{O}$ ), IVM was repeated, focused on the expiratory plateau. The IPP of $18 \mathrm{cmH}_{2} \mathrm{O}$ during IVM was chosen because this is the maximum IPP, which avoids ventilator-induced lung injury (VILI) when applied for a short time. The rabbit was regularly ventilated for 5 min between acquisitions of IVM images. Limited by the sample rate of the OCT system, the acquisition of the 3 -D image stacks $(320 \times 320 \times 512$ pixels/approximately $1.3 \times 1.3 \times 2 \mu \mathrm{m}^{3}$ ) takes about $8.5 \mathrm{~s}$. For data capture, the ventilation was stopped at a constant positive airway pressure (CPAP) of $12 \mathrm{cmH}_{2} \mathrm{O}$ applied for, at most, $10 \mathrm{~s}$ (see Fig. 1). OCT imaging was only carried out at a CPAP of $12 \mathrm{cmH}_{2} \mathrm{O}$ to demonstrate the feasibility of OCT imaging in this model. The 3-D reconstructions of the alveolar structures were carried out by the Amira 4.1 software (Visage Imaging, San Diego, California).

\section{Results}

Briefly, the model was very stable for the time of investigation, and we detected no negative effects in hemodynamic $(\mathrm{MAP}=80 \pm 8 \mathrm{mmHg})$, blood gases $\left(\mathrm{pO}_{2}=86 \pm 12 \mathrm{mmHg}\right.$, $\mathrm{paCO}_{2}=28 \pm 5 \mathrm{mmHg}$ ), and electrocardiogram (heartbeat $=173 \pm 15 \mathrm{bpm}$ ) after investigation. In this feasibility study, the experiments were stopped after $3 \mathrm{~h}$. Also, no edema or inflammation caused by surgical preparation was observed.

Figure 2 shows the prepared thorax window (TW) (approximately $10 \times 5 \mathrm{~mm}^{2}$ ), which was used for imaging alveolar lung tissue in vivo. The photograph of the ribs (R) and thorax window (TW) was taken from the intrathoracal side and was taken at the end of the experiment after resection of the tissue. In this figure, the undamaged parietal pleura $(\mathrm{P})$ can be recognized. Figures 2(b) and 2(c) present that we are able to image identical alveoli during the whole ventilation cycle. The identical alveoli are denoted in the IVM image acquired in the expiratory phase as well as in the inspiratory phase by labels $a, b, c$, and $d$. The alveolar area marginally expanded approximately $7 \pm 5 \%(\mathrm{n}=15)$ at $18 \mathrm{cmH}_{2} \mathrm{O}$, compared to $2 \mathrm{cmH}_{2} \mathrm{O}$ ventilation pressure. Figure 3 demonstrates that the described animal model is also suitable for 3-D OCT imaging. In Fig. 3(a), the intact parietal pleura (P) with 

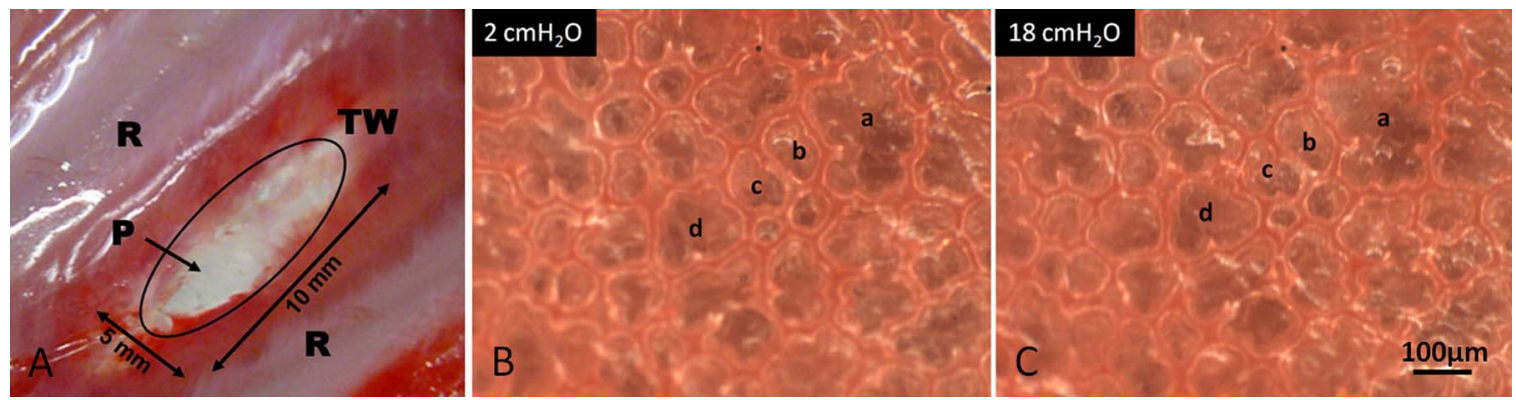

Fig. 2 (a) Prepared thorax window (TW/black ellipse) between the third and fourth rib (R) photographed after dissection from the intrathoracal side showing the transparent uninjured parietal pleura (P). (b) and (c) are IVM images acquired through the uninjured parietal pleura in the expiratory [(b) $2 \mathrm{cmH}_{2} \mathrm{O}$ ] and inspiratory [(c) $18 \mathrm{cmH}_{2} \mathrm{O}$ ] plateau phase. The identical alveoli labeled a, b, c, and d can be detected in both images. An expansion of approximately $7 \%$ of the alveolar area can be recognized.

approximately $50 \mu \mathrm{m}$ thickness can be seen on top of the lung surface in the 2-D cross sectional OCT images, acquired with an applied CPAP of $12 \mathrm{cmH}_{2} \mathrm{O}$. In the 2-D cross sectional OCT images, single alveoli (A) and the border between two lobes (B) of the lung can be recognized. Figure 3(b) shows a 2-D OCT enface image of subpleural lung parenchyma extracted from the acquired 3-D OCT image stack. The enface image represents the subpleural alveolar tissue in a $45 \mu \mathrm{m}$ depth beneath the pleura, and was used to quantify alveolar areas. ${ }^{8}$ The mean of the alveoli area is $7.015 \mu \mathrm{m}^{2}$ with a minimum of $1.984 \mu \mathrm{m}^{2}$ and a maximum of $20.368 \mu \mathrm{m}^{2}$. The 3 -D reconstruction of a single alveolar structure for a CPAP of $12 \mathrm{cmH}_{2} \mathrm{O}$ can be seen in Fig. 3(c). Furthermore, the 3-D reconstruction shown in Fig. 3 features a triangle mesh, which can be used as a basis for 3-D numerical models of the lung.

\section{Discussion and Conclusion}

We present a novel method for thorax window preparation for optical imaging that, unlike other techniques, ${ }^{9}$ can avoid the induction of pneumothorax during preparation and investigation. The described innovative preparation technique allows that when the parietal pleura is retained as a transparent membrane, IVM and 3-D OCT can be performed with an intact chest. This technique allows for tracking the identical alveoli during the whole ventilation cycle in healthy lungs. Furthermore, we have shown that OCT is highly suitable for 3-D in vivo imaging of subpleural alveolar structures within a resolution of less than $10 \mu \mathrm{m}$. Other imaging modalities, like computed tomography (CT) or magnetic resonance tomography (MRT), do not offer images at such high resolution. In contrast to techniques where cover glasses are placed on the surface of the lung for 2-D IVM imaging, ${ }^{11}$ we have demonstrated a contact-free measurement without irritation of alveolar dynamics during ventilation. Additionally, it could be shown that a 3-D reconstruction based on 3-D OCT datasets is possible. The quantification of the alveolar areas in the IVM images results in an expansion of alveolar areas. This detected increase in alveolar area with increasing ventilation pressure is confirmed by other studies. ${ }^{8,9}$ The handicap of this model for alveolar imaging is the limited observation area including only a small number of alveoli. Also, the investigated region has to be on the upper lobe, because the dislocation of the inferior lobe is much higher during the ventilation cycle. By

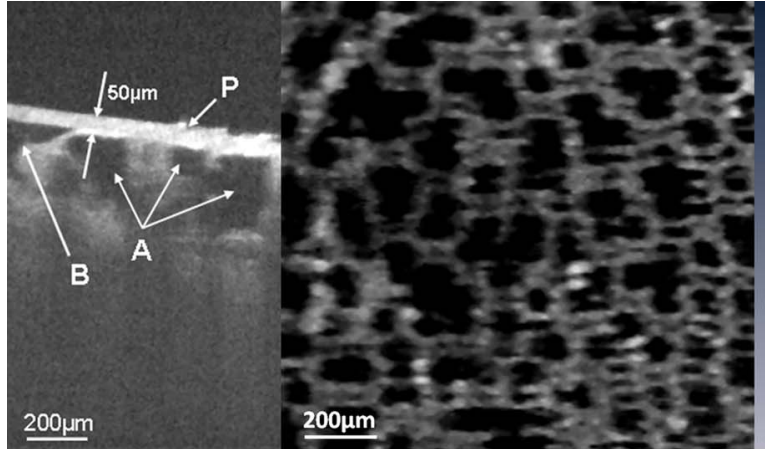

(a) (b)

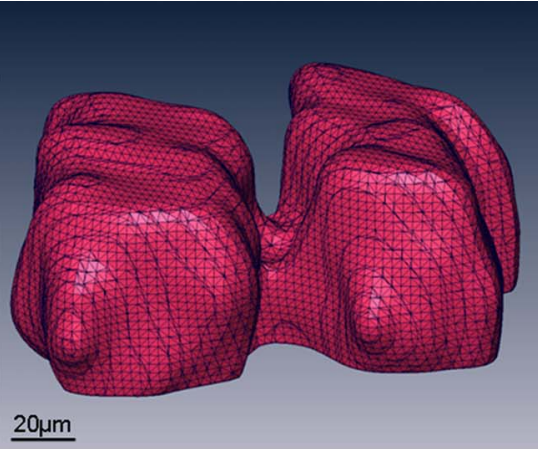

(c)

Fig. 3 (a) Single alveoli (A) and the uninjured parietal pleura (P) with a thickness of approximately $50 \mu \mathrm{m}$ are recognizable in the 2-D cross sectional OCT image of the investigated area. The image was despeckled by a median filter. The image was acquired in the inspiratory plateau with IPP of $12 \mathrm{cmH}_{2} \mathrm{O}$. The border (B) of the upper lobe of the lung is demarked. (b) OCT enface image extracted from the 3-D OCT image stack. The enface image shows the lung parenchyma in a depth of about $45 \mu \mathrm{m}$ beneath and parallel to the pleura. (c) A 3-D reconstruction of single subpleural alveoli at a constant airway pressure of $12 \mathrm{cmH}_{2} \mathrm{O}$ is shown. The alveolar structures are bordered at the upper flat side by the visceral pleura. At the lower side, the reconstruction is limited by the decreasing depth information, what can be seen in the cross sectional OCT image in (a), too. 
gating the 3-D OCT measurement to the inspiratory and/or expiratory plateau, the 3-D OCT image stack can be acquired during ventilation without applying CPAP, as we have previously demonstrated. ${ }^{9}$ A gating to the heartbeat is also possible to minimize moving artifacts. In further studies, we will characterize the alveolar volume change during artificial ventilation in healthy and injured lungs. Additionally, high speed OCT systems allow OCT Doppler measurements, ${ }^{12,13}$ providing blood flow and blood velocity measurements in the arterioles and investigation of the vascular function in healthy and injured lungs without opening the pleura. The 3-D OCT imaging technique in combination with the described animal model might improve knowledge about alveolar geometry and vascular function during ventilation in an in vivo situation. We conclude that dynamic data on alveolar volume change acquired by OCT might provide the basis for the development of numerical models of the lung, by using the surface mesh obtained by 3-D reconstruction of the 3-D OCT data of fine alveolar structures. As shown, OCT overestimates the alveolar walls and underestimates the air-filled alveolar space. ${ }^{14}$ This fact has to be considered using 3-D OCT data as input for numerical models.

\section{Acknowledgment}

This project was supported by the German Research Foundation (DFG) "Protective Artificial Respiration" (PAR)-KO 1814/6-1.

\section{References}

1. J. A. Frank and M. A. Matthay, "Science review: mechanisms of ventilator-induced injury," Crit. Care Med. 7, 233-241 (2003).

2. The Acute Respiratory Distress Syndrome Network, "Ventilation with lower tidal volumes as compared with traditional tidal volumes for acute lung injury and the acute respiratory distress syndrome," $N$. Engl. J. Med. 342, 1301-1308 (2000).

3. H. Kitaoka, G. F. Nieman, Y. Fujino, D. Carney, J. DiRocco, and I. Kawase, "A 4-dimensional model of the alveolar structure," $J$.
Physiol. Sci. 57, 175-185 (2007).

4. D. Huang, E. A. Swanson, C. P. Lin, J. S. Schuman, W. G. Stinson, W. Chang, M. R. Hee, T. Flotte, K. Gregory, C. A. Puliafito, and J. G. Fujimoto, "Optical coherence tomography," Science 254, 1178-1181 (1991).

5. G. Haeusler and M. W. Lindner, "Coherence radar and spectral radar-new tools for dermatological diagnosis," J. Biomed. Opt. 3, 21-31 (1998).

6. S. Meissner, G. Mueller, J. Walther, H. Morawietz, and E. Koch, "In vivo Fourier domain optical coherence tomography as new tool for investigation of vasodynamics in the mouse model," J. Biomed. Opt. 14(3), 34027 (2009).

7. A. Popp, M. Wendel, L. Knels, T. Koch, and E. Koch, "Imaging of the three-dimensional alveolar structure and the alveolar mechanics of a ventilated and perfused isolated rabbit lung with Fourier transform optical coherence tomography," J. Biomed. Opt. 11(1), 014015 (2006).

8. S. Meissner, L. Knels, A. Krüger, T. Koch, and E. Koch, "Simultaneous 3D optical coherence tomography and intravital microscopy for imaging subpleural pulmonary alveoli in isolated rabbit lungs," $J$. Biomed. Opt. 14(5), 054020 (2009).

9. M. Mertens, A. Tabuchi, S. Meissner, A. Krueger, U. Kertzscher, A. R. Pries, K. Affeld, A. S. Slutsky, E. Koch, and W. M. Kuebler, "Alveolar dynamics in acute lung injury: heterogeneous distension rather than cyclic recruitment," Crit. Care Med. 37(9) 2604-2611 (2009).

10. J. T. Wearn, A. C. Ernstene, A. W. Bromer, J. S. Barr, W. J. German, and J. Zschiesche, "The normal behaviour of the pulmonary blood vessels with oberservation on the intermittence of the flow of blood in the arterioles and capillaries," Am. J. Physiol. 109(2), 236 (1934).

11. J. D. DiRocco, D. E. Carney, and G. F. Nieman, "Correlation between alveolar recruitment/derecruitment and inflection points on the pressure-volume curve," Intensive Care Med. 33(7), 1114-1116 (2007).

12. J. Walther, G. Mueller, H. Morawietz, and E. Koch, "Analysis of in vitro and in vivo bidirectional flow velocities by phase-resolved Doppler Fourier-domain OCT," Sens. Actuators, A 156(1) 14-21 (2009).

13. P. Cimalla, J. Walther, M. Mehner, M. Cuevas, and E. Koch, "Simultaneous dual-band optical coherence tomography in the spectral domain for high resolution in vivo imaging," Opt. Express 17(22), 19486-19500 (2009).

14. S. Meissner, L. Knels, and E. Koch, "Improved 3D Fourier domain optical coherence tomography by index matching in alveolar structures," J. Biomed. Opt. 14(6), 064037 (2009). 